\title{
La transposición didáctica del funcionamiento hidrostático de un órgano complejo: la vejiga natatoria de los peces
}

\author{
J. Carrasquer Zamora; A. Ponz Miranda; $\mathbf{M}^{\mathrm{a}}$. V. \\ Álvarez Sevilla; C. Lázaro Peinado; J. Bujeda Gómez \\ Departamento de Didáctica de las Ciencias Experimentales \\ Universidad de Zaragoza
}

Resumen: La vejiga natatoria de los peces y en concreto su función hidrostática son habitualmente utilizadas en diversos niveles educativos obligatorios como ejemplo para que el alumnado generalice el principio de Arquímedes; asimismo se hacen analogismos con el funcionamiento del ludión, el submarino tradicional o el globo aerostático; también se presenta como un logro adaptativo de los seres vivos. En la mayoría de los casos se ejemplifica sobre desplazamientos verticales, dando nula, o menor importancia a su función de flotación neutra o de estabilidad en un nivel determinado del agua. La aparente sencillez de funcionamiento de la vejiga natatoria equiparándola a un globo o flotador, puede ocasionar una distorsión en el aprendizaje introduciendo concepciones superadas por la comunidad científica.

Palabras clave: Historia de la Ciencia, vejiga natatoria, enseñanza, obstáculos en el aprendizaje, lenguaje científico.

Abstract: The swim bladder of fish, particularly its hydrostatic function, are commonly used in various compulsory educational levels as an example to generalize the Archimedes' principle among students. Analogisms are also made with the Ludion, the functioning of the traditional submarine, or the aerostatic balloon. The swim bladder is considered an adaptive achievement of living beings as well. In most cases, it is exemplified with vertical displacements, giving no or less importance to the function of neutral buoyancy, or stability at a certain level of water. The apparent simplicity of the swim bladder operation when it is just compared with a balloon or float, can lead to distortion in student's knowledge by introducing learning concepts already overtaken by the scientific community.

Keywords: History of Science, swim bladder, teaching, learning obstacles, scientific language.

(Fecha de recepción: junio, 2013, y de aceptación: septiembre, 2013)

DOI: 10.7203/DCES.27.3005

* El Dpto. de Ciencia, Tecnología y Universidad del Gobierno de Aragón y el Fondo Social Europeo financian el Grupo Consolidado de Investigación Aplicada Beagle (BOA, 9/5/2008). 


\section{Introducción}

"El señor Juan, de El Bichar (Isla de Coche, Venezuela), me contó que los pescadores de la isla trabajaban con sus pequeñas barcas capturando lisas y pargos. También pescaban de forma comunitaria, cuando era necesario, para capturar grandes cardúmenes de pescado ya que era obligado sumar brazos, barcas, motores y metros de redes para capturar las sardinas o los grandes carites. Me explicó que cuando desde los acantilados de Guamache, también en la Isla de Coche, veían que abundantes burbujas emergían del mar, sabían que un cardumen de sardinas estaba ya cerca de la superficie; era hora de aunar materiales y esfuerzos para conseguir una fructífera jornada de pesca." (J. Carrasquer, com. pers.).

Este relato le sirvió a un joven biólogo para que su idea acerca del funcionamiento de la vejiga natatoria de los peces entrara en conflicto con lo que él pensaba: no aumentaba la cantidad de gas en la vejiga natatoria cuando subía el pez, sino que disminuía, el agua burbujeaba cuando los peces se desplazaban pausadamente hacia la superficie. Se desbarató una creencia equivocada, pero se mantuvieron otras también erróneas, como que la masa del aire contenido en la vejiga natatoria era representativa para modificar sustancialmente la densidad del pez.

En ocasiones la simplicidad con la que se trabaja en las aulas de diversos niveles educativos el funcionamiento físico-biológico de la vejiga natatoria (ejemplo utilizado para ayudar a la comprensión del principio de Arquímedes junto con el ludión, el globo aerostático, el submarino o el molusco Nautilus) y la consiguiente inferencia de su fisiología y función en los peces, ocasiona una construcción intelectual no acorde a los avances en el conocimiento (Morales, Rey y Hernández, 2006).

En el aula, el profesorado tiende a ser menos riguroso en el lenguaje utilizado en razón de su carácter efímero y sin embargo, el lenguaje, es un factor fundamental en el aprendizaje del alumnado. El diálogo entre profesores y alumnos y entre éstos, estimula las conversaciones consiguiendo de esa manera que se mejore y amplíe su capacidad intelectual, su comprensión, su lenguaje, así como su vocabulario especializado. Pero no solamente desde un punto de vista cognitivo, sino que la utilización del discurso en el aula se considera una herramienta útil para alcanzar una sociedad más democrática y saludable (Cazden, 2003; Alexander, 2008; Jiménez-Aleixandre, 2011). Si bien la interpretación y construcción de los significados de acontecimientos observados es personal, puede estar muy influenciada por las explicaciones de otras personas, por lo que es importante la verbalización e intercambio de razonamientos. Por otra parte mediante el discurso se afianzan los contenidos necesarios y el alumnado podrá construir mejores argumentos para la defensa de sus opiniones y para valorar la pertinencia de las analogías utilizadas en el proceso de enseñanza.

En este trabajo se presenta un breve resumen de la evolución histórica del conocimiento científico acerca de la función de la vejiga natatoria de los peces 
y los hitos fundamentales en el avance de la clarificación de su función hidrostática. También se sugiere la utilización de la historia de la ciencia como recurso didáctico, en el intento de que el alumnado mejore y amplíe la visión de la naturaleza de la ciencia (Solomon, Duveen y Scot, 1992; Abd-El-Khalick y Lederman, 2000) y su posible utilización en las aulas de niveles superiores de enseñanza no universitaria y en las de estudios universitarios de formación de docentes, como un problema de indagación y utilización del lenguaje discursivo, permitiendo la integración de contenidos de física y de biología.

Desde el punto de vista biológico el funcionamiento y función de este órgano se puede utilizar como recurso de aprendizaje tanto desde el punto de vista anatómico en los primeros cursos de niveles universitarios de titulaciones relacionadas con los seres vivos, veterinaria, ciencias ambientales, biología, ..., como en los contenidos relacionados con la diversidad las adaptaciones de los seres vivos en los mismos niveles o inferiores. Asimismo el recurso puede ser útil en el máster de formación de profesorado y en el grado de magisterio como planteamiento de problemas de indagación. Especialmente interesante desde el punto de vista docente son las variables físicas que interactúan en su funcionamiento, al fin y al cabo fueron las que provocaron el estudio del funcionamiento de la vejiga natatoria desde la perspectiva mecanicista cartesiana. Algunos de estos aspectos se tratan en Carrasquer, Ponz, Álvarez y Gil (2013).

\section{Hitos históricos relevantes acerca de la función hidrostática de la vejiga natatoria}

La vejiga natatoria y en concreto la misión que cumple en los peces así como su funcionamiento han llamado la atención de los filósofos e investigadores a lo largo de toda la historia.

Aristóteles (Tower, 1902) señalaba a este órgano como el responsable de parte de los sonidos generados por los peces. Desde la antigüedad se identificaban los movimientos verticales de los peces con el principio de Arquímedes y posteriormente con el diablillo cartesiano, cuando este aparato se popularizó a partir de mediados del siglo XVII. En realidad fue la vejiga natatoria la que de alguna manera inspiró la construcción del ludión. En los intentos de Galileo por encontrar un cuerpo sólido, de una densidad similar a la del agua que pudiese servir para la fabricación de termoscopios, una de sus referencias fueron los peces; éstos podían quedarse inmóviles en un punto determinado del agua, por lo que su densidad tenía que ser la misma. ¿Por qué no intentar conseguir ese cuerpo sólido de manera que al variar la temperatura del agua, suba o baje, en función de la densidad del líquido? Galileo en palabras de Salviati, Sagredo y Simplicio, relata sus intentos con una bola de cristal hueca, levemente perforada utilizando como líquido para sus pruebas una mezcla de agua con vino tinto. Dice Salviati, que los peces son capaces de mantenerse en reposo en cualquier lugar y momento, utilizando su vejiga, que está comunicada con un estrecho tubo con la boca. Sagredo cita la 
analogía de la bola de cera que se mantiene en equilibrio en el lugar del recipiente de agua que se le coloque. Salviati responde con el diseño de una analogía de la vejiga, Si lleno con agua una bola de cristal, que tiene un agujero estrecho, del grosor de una hebra de paja y casi llena la vuelvo con la boca hacia abajo [...]. (Galileo, 1638, 71-72).

Desde este momento histórico, antes de que Magiotti (1648) haya explicado el funcionamiento del scherzi y de que se haya utilizado el nombre de diablillo Cartesiano o ludión, ya se puede considerar que se realiza la primera analogía entre él y la vejiga natatoria de los peces, porque éstos son puestos como ejemplo de que la estabilidad en un nivel determinado de un cuerpo sólido en el interior del agua podía ser lograda.

Una de las primeras analogías conocidas, para comprender el funcionamiento de la vejiga natatoria es la utilizada por A.I. ${ }^{1} \&$ Boyle. Apoyándose en las proposiciones de Arquímedes y utilizando como ejemplo lo que sucede con una burbuja de aire que sube en el interior del agua, sugieren que al elevarse el pez y disminuir la presión del agua aumenta el tamaño de su vejiga; al descender el pez, aumenta la presión y la vejiga disminuye.

Como posible demostración de la conjetura describen una propuesta experimental sugerida por Robert Boyle para que se pueda determinar si la hipótesis de la variación del tamaño de la vejiga y del pez debida a la presión del agua, era correcta. El experimento propuesto consistía en utilizar un recipiente con un cuello largo, y teniéndolo casi lleno de agua, poner en él un pez vivo de talla conveniente, es decir el más grande que pudiera entrar, por ejemplo una perca o similar; luego hacer el cuello del recipiente tan delgado como se pudiese y rellenar de agua. Si al sumergirse el pez en lo más profundo del recipiente el nivel del agua baja bajase se podría inferir que la vejiga del pez había disminuido y al contrario (A.I. \& Boyle, $1675,311)$. En este trabajo no se concreta lo que durante los años posteriores será motivo de polémica, ¿qué sucede?, el pez sube y como consecuencia de pérdida de presión, aumenta el tamaño de la vejiga y por lo tanto de su cuerpo, o bien el pez aumenta el tamaño por sus medios, musculares o captando gases y por lo tanto se eleva.

Borelli (1680), defiende una posición similar a Boyle pero además afirma que la vejiga aumenta o disminuye por acción de músculos internos o externos del pez. Este aumento de la vejiga se vería reflejado también en un aumento o disminución del volumen del pez; al aumentar de volumen disminuye su peso específico por lo que sube. Misma masa, para más volumen.

Borelli sugiere otra analogía (Figura I). Propone una demostración con una syringae, jeringa $\mathrm{CAB}$ (los dos dibujos centrales). En el cilindro AB se introduce el émbolo CD saliendo el aire por el orificio M que se cierra una vez que

\footnotetext{
${ }^{1}$ A.I.: [= Anonymous Individual]. Kassler (2004, 26, 223), defiende la idea de que este autor anónimo es Francis North, barón de Guilford.
} 
Figura I. Representación de la modificación del tamaño del pez y su vejiga según la profundidad. Analogía entre la variación de la densidad del pez y de un cilindro con su émbolo.
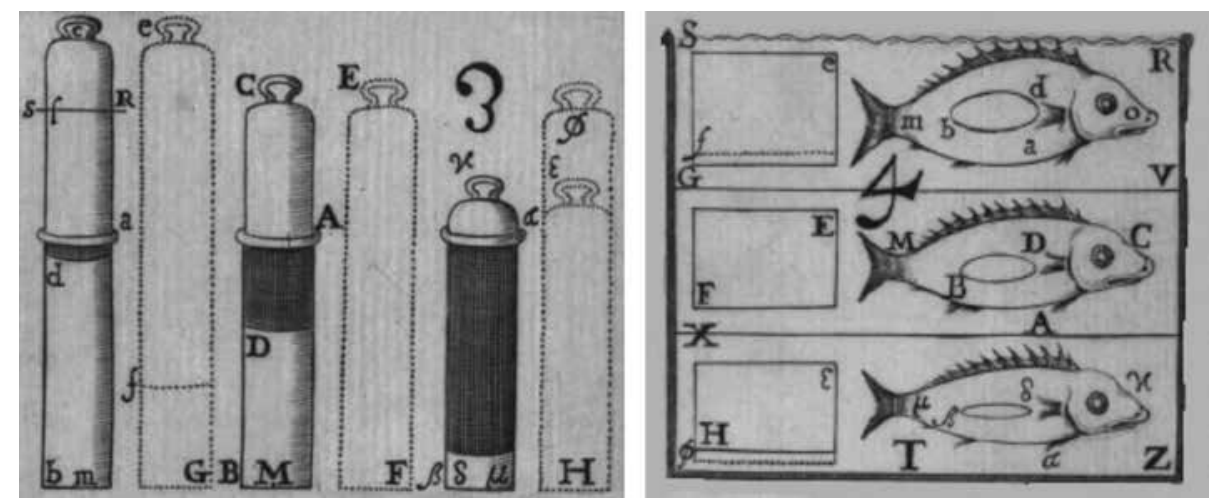

Fuente: BORELLI (1680, tab. XIV, figuras 3- 4).

se ha terminado la operación (el espacio sombreado corresponde a la parte del embolo introducida en el cilindro); el volumen total es EF y el aire en el interior de la syringae está a presión normal, DB. Análogamente el pez CAM tiene un volumen de aire en su interior BD (en el dibujo de Borelli, representado en el centro de la pecera). Si se estira del émbolo, situación representada en cab (los dos dibujos de la izquierda), la misma cantidad de aire ocupará el espacio db y el volumen de la siringae será cb, misma masa para un mayor volumen; similar situación representada en el pez cam y su vejiga bd (pez superior). Asimismo la situación contraria en los dos dibujos de la jeringa de la derecha y del pez de la parte inferior (Borelli, 1680, 336).

Artedi (1738, 34 y ss.) describe especies sin vejiga, con vejiga conectada mediante un canal, ductus pneumaticus, con el tubo digestivo y otras especies con la vejiga aislada.

Delaroche (1809) aporta la hipótesis de que la vejiga natatoria no cumple la misión de aumentar o disminuir el peso específico del pez para bajar o subir, sino que le sirve para igualarlo al del agua para así mantener el nivel de equilibrio. Cuvier, Lacépède y Vauquelin, profesores valoradores de la revista Anales de Historia Natural de Paris, a pesar de reconocer el mérito de la investigación de Delaroche no aceptan esta idea, apoyando lo dicho por Borelli y publican en el mismo número una réplica al trabajo de Delaroche (Cuvier, 1809, 373).

Weber (1820, 48-51) describe las conexiones de ciertos peces entre la vejiga y el aparato acústico-laberíntico.

Investigaciones posteriores llevadas a cabo por diversos autores (Moreau, 
1876; Charbonnel-Salle, 1887; Guyénot, 1909) ratificarán la idea de que el pez tiene un plano de estabilidad en el que su densidad es la misma que la del agua y en ella tiene una voluntaria y perfecta movilidad horizontal, apoyando la teoría de Delaroche.

Moreau diseña diversas experiencias que lleva a cabo para demostrar sus hipótesis, algunas de ellas contestadas por otros investigadores. En cualquier caso aporta una serie de principios importantes acerca de la vejiga natatoria, mejorando lo dicho hasta la fecha. Además de insistir en la importante idea de Delaroche, indica que la vejiga es un órgano sustancialmente peligroso para los peces que lo poseen, afirmando que su función es de equilibrio y que es un inconveniente para los desplazamientos verticales, poniendo en duda su eficacia evolutiva.

La capacidad de desplazamientos hacia arriba o hacia abajo se la asigna a la fuerza muscular del cuerpo y a las aletas; como consecuencia de la variación de la presión en los desplazamientos verticales, el pez por tanto, tiene que acomodar el tamaño de la vejiga variando proporcionalmente la cantidad de gases de su interior, para mantener la presión en el interior de la vejiga constante. Dado que al elevarse el pez sufrirá una disminución de presión en la vejiga y aumentará de tamaño, pudiendo romperse, por lo que el pez tendrá que disminuir la cantidad de gases de ella. El objetivo es que presión del gas en el interior de la vejiga sea constante.

Es decir el tamaño de la vejiga ya no depende de un esfuerzo muscular de la vejiga y de las paredes musculares del pez, sino que depende de la presión, la del agua que ejerce sobre el propio pez y la del gas del interior de la vejiga que ejerce sobre ella.

Moreau también afirma que el control de la cantidad de gas dentro de la vejiga es un proceso más lento de lo que se creía, pero que se conseguía habitualmente. Esta lentitud tiene la excepción de los peces que tienen la vejiga comunicada con un conducto al tubo digestivo (Fisóstomos); en este caso la expulsión de los gases en el proceso de ascensión puede ser rápida. En estos peces en el caso de los descensos se comportan igual que los que no tienen el canal neumático.

Hall en 1924, reafirma que la función primaria de la vejiga es la hidrostática y que permite al pez mantener su estabilidad independientemente del cambio en las condiciones externas de su ambiente.

Guillemin en su tratado de física llama la atención acerca de lo poco oportuno del nombre vejiga natatoria, una vez demostrado que la función del órgano no es facilitar los desplazamientos verticales como se creía sino permanecer estable en un nivel. El autor al describir analogías para reforzar la comprensión del Principio de Arquímedes dice, Los peces suben y bajan á su albedrío en el seno del agua. Largo tiempo se ha creído, y nosotros mismos lo hemos repetido siguiendo el parecer de muchos sabios físicos ó fisiólogos, que lo que hace posible estos diferentes movimientos es la facultad que tienen estos animales de comprimir ó hinchar una especie de bolsa elástica llena de 
aire, colocada en el abdomen. [...]. Pero en la actualidad parece demostrado que no son tales las funciones de la vejiga natatoria. [...]. En realidad la vejiga se dilata cuando el pez se eleva sobre cierto nivel en el cual se halla en equilibrio, gracias á la igualdad de su densidad con el agua. [...]. Pero si el pez desciende bajo el nivel de equilibrio, como la presión exterior aumenta, la vejiga se contrae, y siendo ya más denso que el agua, caería hasta el fondo si permaneciera inmóvil; por lo cual se remonta de nuevo merced á sus esfuerzos musculares. (Guillemin, 1893, 75).

Aguilar ofrece una descripción acertada de lo que se debe saber para entender la función hidrostática de la vejiga natatoria, El papel de la vejiga natatoria es muy limitado: ayuda al pez a permanecer a una profundidad determinada, que depende de la densidad del pez. Cuando el pez, moviendo las aletas, baja su cuerpo experimenta una presión exterior mayor por parte del agua y se contrae comprimiendo la vejiga. De esta forma el peso del agua que desaloja disminuye y el pez tiende a descender más. Lo mismo ocurre, pero en sentido contrario, cuando el pez se eleva moviendo las aletas. Al disminuir la presión exterior la vejiga se distiende y más rápida se hace la ascensión. El pez no puede oponerse a esto comprimiendo la vejiga natatoria por la sencilla razón de que las paredes de ésta carecen de fibras musculares que le permitan variar el volumen activamente. (Aguilar, 2000, 223)

Estos avances en el conocimiento científico tienen distinto reflejo según los textos que se utilicen, quedando confusos o también con inclusión de algu- na incorrección. Por ejemplo en Cromer, Esta cavidad de paredes delgadas se llena con una mezcla de oxígeno y nitrógeno obtenida de la sangre. Variando la cantidad de gas de la cavidad, puede variarse el volumen del pez sin modificar su masa, lo que le permite ajustar su densidad. Para flotar suspendido en el agua, ha de ajustar su densidad a la del agua ambiente (Cromer, 1996, 164-5).

En este párrafo de Cromer se ha de interpretar que los gases pasan de la sangre a la vejiga o viceversa, sin aumentar o disminuir su cantidad total en el cuerpo del pez. Sin embargo no contempla la expulsión de gases a través del tubo digestivo (peces fisóstomos).

Miscelánea física. Los peces. Tanto los peces, como muchos animales marinos, aprovechan el principio de Arquímedes para hundirse o salir a al superficie. Los tejidos de los peces son más densos que el agua, sin embargo, los peces teleósteos, por ejemplo, poseen una cavidad interior, llamada vejiga natatoria, que se llena de gas y permite que su densidad sea prácticamente igual a la del agua, lo que se les permite salir más fácilmente a la superficie. Como la vejiga natatoria es flexible, el pez puede variar su densidad para hundirse a salir a flote. (Sandoval, 2000, 69).

En el caso de Sandoval queda reflejado el error más habitual, el de pensar que el pez consigue mayor tamaño de la vejiga, aumentando la cantidad de gas y se olvida de la influencia de la presión hidrostática que es realmente la que provoca el aumento o disminución del tamaño. 


\section{La vejiga natatoria, un órgano de estabilidad}

La vejiga de gases es un órgano que no está presente en todos los peces, es de morfología muy variada y de fisiología compleja; su función tardó muchos siglos en clarificarse. Desde Aristóteles hasta el siglo XIX surgieron diversas teorías y propuestas acerca de su función, que se fueron matizando gracias a un proceso experimental que puede servir de ejemplo a los estudiantes, siendo un modelo de la manera en que se construyen los conocimientos científicos.

También, en esta ocasión, la utilización del lenguaje se manifiesta como un factor clave en la construcción del conocimiento (Sutton, 2003). Los sucesos en los que intervienen diversas variables, si éstas no son explicitadas y verbalizadas adecuadamente, pueden ser de difícil comprensión para los aprendices, impidiendo la construcción de cómo cada una de ellas influye en el acontecimiento observado y por lo tanto de su correcto aprendizaje.

En el caso de la estabilidad del pez en un nivel del agua determinado y por lo tanto de su igual densidad, son variables a tener en cuenta como en cualquier otro cuerpo la masa y el volumen. Cuando se atribuye, por ejemplo, la disminución de la densidad del pez y por lo tanto su elevación en el agua, al aumento del tamaño de la vejiga natatoria, se está dejando sin concretar a qué es debida esa disminución de la densidad, si a un aumento del tamaño del pez o a una disminución de su masa.

Al contrario de lo que sucede en la analogía propuesta por Borelli (1680), apoyada por dibujos que no dejan lugar a dudas, de que es el aumento del volumen del pez el responsable de la disminución de su densidad, parece que en las representaciones gráficas que se utilizan habitualmente en la actualidad (Figura II), ninguna de las dos variables es tenida en cuenta, dado que la masa del pez, en todo caso aumenta porque hay más gases, por lo que la densidad también lo hace. No se tiene en consideración el aumento del tamaño del pez, que se interpreta como una caja rígida, siendo únicamente aceptado el aumento del tamaño de la vejiga en el interior del cuerpo del pez.

\section{Figura II. Representación animada del funcionamiento de la vejiga natatoria de un pez.}
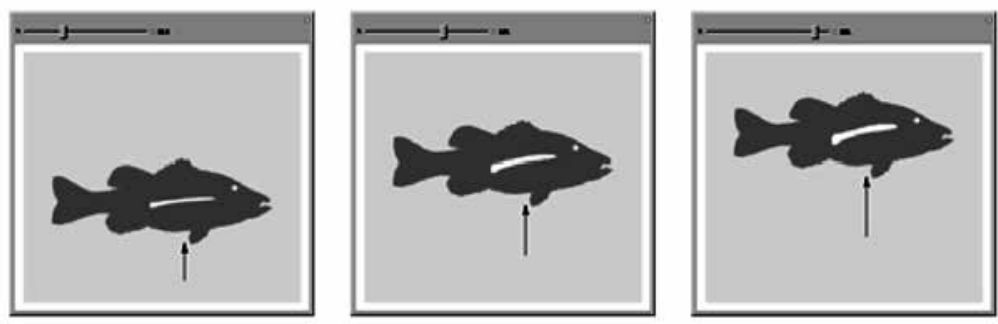

Fuente: ZELENY (2012) 


\section{Representación del funcionamiento de la vejiga natatoria de un pez.}

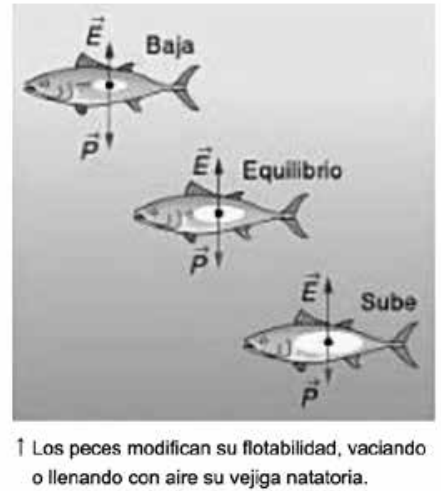

Fuente: BARRIO, ANDRÉS y ANTÓN, (2008).

Otra variable imprescindible que se debiera considerar para comprender el funcionamiento de la vejiga natatoria es la presión que sufre el pez en el interior del agua y cómo ésta puede influir en el tamaño de la vejiga y del pez, siendo la variable fundamental para construir este modelo que se utiliza como analogía. Paradójicamente, en los ejemplos propuestos habitualmente para la comprensión del Principio de Arquímedes, que es el que se quiere reforzar, esta variable no es relevante.

Tal como se ha indicado anteriormente en el razonamiento utilizado para explicar la función de la vejiga natatoria se pone de manifiesto que habitualmente se dice que es un órgano de flotación y no que es un órgano necesario para alcanzar la estabilidad del pez en un nivel de agua determinado. Por esto es preciso tener en consideración cuál es la significación que para el alumnado tienen los conceptos utilizados ya que serán los que ellos empleen para construir su aprendizaje.

La palabra vejiga llena de gases, unida a la de flotación, conduce de forma mayoritaria a unos conceptos conocidos desde la infancia, globo y flotador. Para el diccionario de la Real Academia Española el verbo flotar (Del fr. flotter), en su primera acepción, "Dicho de un cuerpo: Sostenerse en la superficie de un líquido"; en su segunda, "Dicho de un cuerpo: Sostenerse en suspensión en un líquido o gas."

La primera acepción de la Academia es la más utilizada para construir nuevos conocimientos o comprender un texto o una conversación y por lo tanto se puede considerar como un obstáculo para la correcta comprensión del fenómeno de la flotabilidad neutra o estabilidad del pez en un nivel del agua determinado. Este obstáculo puede potenciarse si se modeliza la vejiga natatoria con un globo externo, adosado al pez.

Las cien primeras entradas del verbo "flotar" en el buscador de Google, en la opción imágenes (14 de junio 2012), el sentido de ellas se reparte de la siguiente manera:

$2 \%$ A. El cuerpo tiende a subir indefinidamente hacia el cielo.

$19 \%$ B. El cuerpo se sostiene suspendido en el aire.

$65 \%$ C. El cuerpo se sostiene en la superficie de un líquido.

$4 \%$ D. El cuerpo se sostiene suspendido en un líquido.

0\% E. El cuerpo se sostiene apoyado en el fondo de un líquido.

$10 \%$ F. Representan imágenes con un sentido figurado. 
Las cien primeras entradas del verbo "to float" en el buscador de Google, en la opción imágenes (14 de junio 2012), el sentido de ellas se reparte de la siguiente manera:

$$
\text { A. } 0 \% \text {; B.- } 18 \% \text {; C.- } 47 \% \text {; D.- } 2 \% \text {; }
$$
E.- $0 \%$; F.- $33 \%$

Preguntado el alumnado de diversos niveles educativos acerca del significado que ellos/as tienen del verbo "flotar" los resultados han sido los siguientes. Se ha utilizado como muestra un grupo de 68 estudiantes de segundo curso del Grado de Magisterio Educación Infantil de (Centro oculto para revisión) 17 alumnos/as de $2^{\circ}$ curso de Bachillerato; 37 alumnos/as de $1^{\circ}$ de Bachillerato; 28 alumnos/as de $4^{\circ}$ curso de E.S.O. Los alumnos de Enseñanzas no universitarias (Centro oculto para revisión)

$2^{\circ}$ curso de Magisterio: 70, $6 \%$ responden eligiendo exclusivamente la opción C.

$2^{\circ}$ curso de Bachillerato: $29,4 \%$ responden eligiendo exclusivamente la opción C. Si se suman los que eligen conjuntamente la opción $\mathrm{C}$ y $\mathrm{B}$, el \% sube al 58,8 .

$1^{\circ}$ curso de Bachillerato: $32,4 \%$ responden eligiendo exclusivamente la opción C. Si se suman los que eligen conjuntamente la opción $\mathrm{C}$ y $\mathrm{B}$, el \% sube al 48,7 .

$4^{\circ}$ curso de E.S.O.: $60,7 \%$ responden eligiendo exclusivamente la opción C. Si se suman los que eligen conjuntamente la opción C y B, el \% sube al 82,1.

De los datos anteriores se puede deducir que existe una correspondencia aceptable entre los datos obtenidos en la consulta del buscador de Google y las contestaciones del alumnado, siendo mayoritaria la significación funcional del verbo flotar para los estudiantes, con la de sostenerse en la superficie de un líquido o en todo caso compartida con la de mantenerse en suspensión en el aire. La significación que en este caso interesa para este trabajo, que es la de mantenerse estable en un nivel en el interior de un líquido, ha sido opción única o compartida con otras, para 32 alumnos $(21,3 \%)$. Es decir este porcentaje es el del alumnado que podría identificar el verbo flotar con la de mantenerse en un líquido, siempre que se matice la utilización del verbo por parte del interlocutor.

Nuevamente, como sucede con otros conceptos, al repasar la evolución histórica del conocimiento del funcionamiento de la vejiga natatoria nos tropezamos con unos obstáculos epistemológicos que se repiten en el aprendizaje individual, si no de una forma totalmente paralela, si con ciertas coincidencias. Además de la dificultad para demostrar las diversas ideas acerca de su funcionamiento y función, existió una gran dificultad para su demostración y también una reticencia manifiesta a su aceptación una vez confirmados (Guyènot, 1909). A pesar de los avances científicos que pusieron de manifiesto el funcionamiento de la vejiga natatoria, los hechos observados acerca de la flotación de globos o vejigas llenas de aire se manifestaban mucho más potentes para construir las explicaciones personales que la existencia de la presión hidrostática y su influencia en los peces y sus órganos.

En la transposición de estos contenidos a las aulas y una vez avanzado el conocimiento complejo de su funcionamiento y puesta de manifiesto su prin- 
cipal función de estabilidad, se publicaron trabajos encaminados a su enseñanza incluyendo los últimos avances en la comprensión de su función (Lapierre, 1925). Pero la vejiga natatoria, su función y funcionamiento no sólo ha sido objeto de estudio directo, sino que también ha sido utilizada como analogía, en niveles de enseñanza obligatoria para explicar otros contenidos como por ejemplo el Principio de Arquímedes tal como se ha indicado anteriormente. Para comprender este último también es habitual utilizar el funcionamiento del ludión por lo que éste y vejiga natatoria suelen ir asociados; asimismo se ejemplifica con el submarino clásico y sus cámaras de aire que se llenan o vacían de agua; o también se encuentra la propuesta de compararla con el compensador de flotabilidad de los submarinistas.

Estas analogías crean confusión, reforzando un conocimiento científico erróneo respecto al funcionamiento y función de la vejiga natatoria de los peces (Carrasquer, Ponz, Álvarez y Gil, 2013).

\section{La transposición didáctica de la función de la vejiga natatoria y su funcionamiento a las aulas de educación primaria y secundaria}

Dada la complejidad del funcionamiento de la vejiga natatoria, puesta de manifiesto por la dificultad histórica para demostrarla y asimismo las reticencias de la comunidad científica para aceptar las nuevas opiniones acerca de su funcionamiento no debe extrañar la dificultad para encontrar una analogía global adecuada que puedan ser utilizada en el ámbito educativo.

La búsqueda de modelos parciales que ayuden a la comprensión del funcionamiento de la vejiga natatoria será la que pueda ayudar a que el estudiante construya el modelo complejo que se pretende. Por ejemplo para trabajar la variación de la densidad puede utilizarse el aumento de tamaño de burbujas o globos al disminuir la presión, manteniendo igual masa (A.I. \& Boyle, 1675) o con el ejemplo de las jeringuillas sugerido por Borelli (1680).

Respecto al otro aspecto fundamental, la estabilidad del cuerpo en un nivel determinado, su desplazamiento vertical y su adecuación al nuevo nivel de estabilidad, aumentando o perdiendo gases, no se ha encontrado analogía adecuada. Ni siquiera, en la actualidad, los globos aerostáticos comerciales permiten la pérdida de gases para mantener un nivel de estabilidad determinado y su autónoma y vertiginosa ascensión termina con la rotura del globo, tal como sucede con la ascensión forzada en los peces.

Uno de los problemas detectados en las analogía utilizadas en las aulas radica en la aceptación por parte de los estudiantes de que un globo hinchado en el interior de un cuerpo, sin modificación del tamaño de éste último, hace disminuir su densidad. Para desbaratar esta idea ampliamente extendida debería trabajarse, por ejemplo con botellas de paredes rígidas con globos en su interior.

Los obstáculos que condicionan la comprensión del funcionamiento de la vejiga natatoria pueden separarse en 
los que tienen que ver con la física y los que relacionan con el propio conocimiento de la anatomía y del funcionamiento fisiológico de la vejiga natatoria.

\section{Físicos}

El Principio de Arquímedes aplicado a un ser vivo cuya densidad varía en sus partes, órganos y tejidos.

La presión atmosférica sigue ejerciéndose en el interior del agua. A ella hay que sumar la presión hidrostática.

Es necesaria una construcción y utilización del significado de los conceptos flotar, flotación, equilibrio.

Conocer las variables que influyen en un proceso es importante: aumentar o disminuir el tamaño no significa necesariamente que haya más o menos gas en su interior. El aumento o disminución de la vejiga puede ser debido al aumento de la presión interior (más gases) o la disminución de la presión exterior (subir y disminuir la presión sobre el pez). Por lo tanto al influir las dos variables, puede haber un aumento del tamaño de la vejiga paralelamente a una disminución de la masa de gas.

El orden de los acontecimientos también es importante. En el caso de la vejiga, primero es subir o bajar y luego aumentar o disminuir el tamaño.

Un mismo efecto puede tener causas diferentes. Si se utiliza la analogía del ludión, efectivamente el efecto es el mismo, a mayor presión, el ludión baja. Pero en el ludión tradicional hay modificación de la masa y no del volumen, mientras que en el pez se modifica el volumen pero no la masa en una cantidad significativa. Por la misma razón la analogía del submarino.

\section{Biológicos}

La voluntariedad de los actos de los animales. El antropocentrismo en el comportamiento animal puede ser apoyado por esta actividad.

Desconocimiento de los distintos tipos de peces en función de si poseen vejiga o no. En el primero de los casos, si esta conectada con el tubo digestivo (Fisóstomos) o no (Fisoclistos). Para el problema planteado no es relevante la forma u distribución de la vejiga en varias cavidades, pero sí, por su influencia en el volumen exterior del pez. Es preciso concretar de qué posibilidad anatómica se está hablando ya que los aspectos fisiológicos varían sustancialmente. Desconocimiento de cómo pueden captar y desprender los gases los peces fisóstomos y fisoclistos, lo que influye directamente en la comprensión de la velocidad a la que se pueden realizar los intercambios. Captación de gases para llenar la vejiga natatoria a través del tubo digestivo. No son relevante para el problema planteado los gases que se recogen en la vejiga natatoria y mediante qué procedimientos fisiológicos, todavía en discusión científica.

La simplicidad en el diseño no ha ser prioritaria respecto a lo que el conocimiento científico dice. Se ha de procurar evitar que la transposición didáctica pueda generar obstáculos nuevos en el aprendizaje.

\section{Conclusión}

Este trabajo aporta un nuevo ejemplo de paralelismo entre la historia de la ciencia y el proceso de enseñanza 
aprendizaje, en el que se pone de manifiesto la persistencia de la ciencia alternativa al pensamiento actualizado de la comunidad científica.

La utilización del ejemplo de la vejiga natatoria de los peces para comprender el Principio de Arquímedes surgió con anterioridad a la explicación de cómo funciona la vejiga. Sin embargo la analogía ha perdurado hasta nuestros días en las aulas, siendo posible que el propio ejemplo, no cuestionado, tenga mucho que ver en que no se entre en conflicto con la ciencia alternativa utilizada para explicar el funcionamiento del órgano de los peces.

La propia complejidad del funcionamiento de la vejiga imposibilita la utilización de una analogía global satisfactoria.

Cuando en el ámbito educativo se habla de vejiga natatoria, o mejor vejiga de gases, habría que hacerlo como un órgano de función hidrostática de estabilidad ayudando a aumentar la significación del concepto de flotabilidad.

\section{Bibliografía}

(Para la consulta de los textos históricos aquí referenciados se recomienda introducir el título de la publicación o los datos de la revista en el buscador Google o bien a través de la página books.google.com/).

ABD-EL-KHALICK, F. y LEDERMAN, N.G. (2000). The influence of history of science courses on students' views of nature of science. Journal of Research in Science Teaching, Volume 37, Issue 10, pp.1057-1095.
DOI: 10.1002/1098-2736(200012)37

:10<1057::ai-tea3>3.0.co;2-c

AGUILAR, M. (2000). Biomecánica: La física y la Fisiología. Madrid, Consejo Superior de Investigaciones Científicas.

A.I., \& BOYLE, R. (1675). A conjeture concerning the bladder of air thaht are found is fishes, comunicated by A.I; And illustrated by an experiment suggested by the honorable Robert Boyle. Philosophical Transactions, 10, pp. 310-311. DOI: 10.1098/ rstl.1675.0016

ALEXANDER, R. (2008). Towards Dialogic Teaching: rethinking classroom talk, $4^{\text {th }}$ edition. Nueva York, Dialogos 2008. En http://www. robinalexander.org.uk

ARTEDI, P. (1738). Ichthyologia sive Opera Omnia de Piscibus. Pars II. Lugduni Batavorum, apud Conradum Wishoff.

BARRIO, J., ANDRÉS, D.M., ANTÓN, J.L. (2008). Física y Química. $4^{\circ}$ E.S.O. Madrid, Editex.

BORELLI, J.A. (1680). De Motu Animalium. Pars Prima. Romae, Ex Typographia Angeli Bernabó.

CARRASQUER, J., PONZ, A., ÁLVAREZ, $M^{a}$.V. Y GIL, $M^{a}$.J. (2013). ¿Es el ludión-vejiga natatoria una analogía adecuada? Comunicación presentada en el IX Congreso internacional sobre investigación en didáctica de las ciencias. 9-12 de septiembre, Girona.

CAZDEN, C. B. (2003). El discurso en el aula: el lenguaje de la enseñanza $y$ del aprendizaje. Barcelona, Paidós. 
CHARBONNEL-SALLE, L. (1887). Sur les fonctions hydrostatiques de la vessie natatorie. Académie des Sciences. 1887 (1) t. 104, pp. 13301333.

CROMER, A. (1996). Física para las ciencias de la vida. Barcelona, Editorial Reverté S.A.

CUVIER, G. (1809). Rapport fait à la classe des sciences physiques et mathématiques, sur le Mémoire de M. Delaroche, relative à la vessie aérienne des poissons. Annales $d u$ Musèum D'Histoire Naturelle. Tomo 14. Paris, G. Dufour et Compagnie Libraires, pp. 165-183.

DELAROCHE, F. (1809). Observations sur la vessie aérienne des poissons. Annales du Musèum D'Histoire Naturelle. Tomo 14. Paris, G. Dufour et Compagnie Libraires, pp.184-217.

GALILEO (1638). Discorsi e Dimostrazioni Matematiche intorno à due Nuove Scienze. In Leida, Appresso gli Elsevirii.

GUILLEMIN, A.V. (1893). El Mundo Físico. Barcelona, Montaner y Simón. GUYÈNOT, É. (1909). Les fonctions de la vessie natatoire des possons Téleostéens. Bulletin Scientifique de la France et de la Belgique, $7^{\mathrm{a}}$ Série, T XLIII. Paris. Pp. 203-299.

HALL, F.G. (1924). The functions of the swimbladder of fishes. Biological Bulletin, vol. $47 \mathrm{n}^{\circ}$ 2, pp. 79-126. DOI: $10.2307 / 1536532$

JIMÉNEZ-ALEIXANDRE, Ma . P. (2011). Argumentación y uso de pruebas: Construcción, evaluación y comunicación de explicaciones en
Biología y Geología, en Didáctica de la Biología y la Geología. Barcelona, Editorial Grao, pp. 129-150.

KASSLER, J.C. (2004). The Beginnings of the Modern Philosophy of Music in England. Aldershot, Ashgate Publishing Ltd.

LAPIERRE, G. (1925). Sciences, Cours moyen et superior, $45^{\mathrm{a}}$ leçon: Le Poisson. Revue de l'Enseignement. Primaire et Primaire Supérieur, $\mathrm{n}^{\circ} 38$, Paris, pp. 595-598.

MAGIOTTI, R. (1648). Renitenza certissima dell'Acqua alla Compressione, Dichiarata con varii scherzi, in occasione d'altri Problemi curiosi. Roma, per Francesco Moneta.

MORALES, CH., REY J., y HERNÁNDEZ M. L. (2006). En Dirección General de Universidades y Editorial Santillana (Ed.), Como pez en el agua. VI Feria de Madrid por la Ciencia 2005. Recuperado en http:// www.madrimasd.org/cienciaysocie$\mathrm{dad} /$ feria/publicaciones/

MOREAU,M.A.(1876).Fonction hydrostatique de la vessie natatoire. Association Française pour l'avancement des sciences, Compte rendu de la $4^{\mathrm{ME}}$ Session, Paris, pp. 77-85.

SANDOVAL, M. (2000). El desarrollo de la Física en Méjico. En Las Ciencias de las Interacciones entre materia y energía. Escuela Normal Superior "Prof. José E. Medrano R". Licenciatura en Educación Secundaria. Plan de Estudios 1999. Programa para la Transformación y el Fortalecimiento Académicos de las Escuelas Normales. Pp. 46-93. México. http:// www.ensech.edu.mx/antologias/par/ 
indices/sem4.htm. (Última consulta, mayo 2012).

SOLOMON, J., DUVEEN, J, y SCOT, L. (1992). Teaching about the nature of science through history: Action research in the classroom. Journal of Research in Science Teaching, Volume 29, Issue 4, pp 409-421. DOI: 10.1002/tea.3660290408

SUTTON, C.R. (2003). Los profesores de ciencias como profesores de lenguaje. Enseñanza de las Ciencias, 21 (1), pp. 21-25.

TOWER, R.,W. (1902). The Gas in the Swim-Bladder of Fishes. Bulletin of the United States Fish Commission,
Washington, $N^{o} X X I, 1901$, pp. 125 130.

WEBER, E.H. (1820). De Aure et Auditu HominisetAnimalium.ParsI,DeAure Animalium Aquatilium. Lipsiae, Apud Gerhandum Pleischeum.

ZELENY, E. (2012). Natatory Bladder of a Fish. En http://demonstrations. wolfram.com/NatatoryBladderOfAFish/. (Última consulta, julio 2013). 
